\title{
Simultaneous determination of naproxen, ibuprofen and diclofenac in wastewater using solid-phase extraction with high performance liquid chromatography
}

\author{
Lawrence Mzukisi Madikizela ${ }^{1,2 *}$ and Luke Chimuka² \\ 'Department of Chemistry, Durban University of Technology, PO Box 1334, Durban, 4000, South Africa \\ ${ }^{2}$ Molecular Sciences Institute, University of Witwatersrand, Private Bag X3, Johannesburg, 2050, South Africa
}

\begin{abstract}
The occurrence and removal efficiency for naproxen, ibuprofen and diclofenac in two of eThekwini Municipality's wastewater treatment plants (WWTPs), Kingsburgh and Umbilo, were investigated. This paper describes a simple method that can be used routinely for the simultaneous determination of such compounds in the influent and effluent of the WWTPs. Target compounds were extracted from wastewater and pre-concentrated using the optimized Oasis MAX solidphase extraction (SPE) method. During SPE, the $\mathrm{pH}$ of wastewater samples was adjusted to 2.5; then $100 \mathrm{~mL}$ of each sample was loaded onto a pre-conditioned cartridge. The SPE cartridge was rinsed with methanol:water (10:90\%, v:v) prior to sequential elution of retained analytes with $2 \mathrm{~mL}$ methanol, followed by $2 \mathrm{~mL}$ methanol and acetic acid (90:10, v:v) and 2 $\mathrm{mL}$ of $2 \%(\mathrm{v}: \mathrm{v})$ formic acid diluted using a mixture of methanol and acetic acid (40:60, v:v). The eluted analytes from the SPE cartridge were quantified using high performance liquid chromatography (HPLC) equipped with photo diode array detection. The analytical method was validated by spiking deionized water with 5 and $50 \mu \mathrm{g} \cdot \mathrm{L}^{-1}$ of target compounds, for which the recovery range of 76 to $98 \%$ was achieved with good precision. The instrument quantification limits obtained were $0.1 \mu \mathrm{g} \cdot \mathrm{L}^{-1}$, for naproxen and $0.4 \mu \mathrm{g} \cdot \mathrm{L}^{-1}$ for both ibuprofen and diclofenac. The detected concentrations for naproxen, ibuprofen and diclofenac in the influent of both WWTPs were in the ranges of $15-20 \mu \mathrm{g} \cdot \mathrm{L}^{-1}, 55-69 \mu \mathrm{g} \cdot \mathrm{L}^{-1}$ and $6.4-16 \mu \mathrm{g} \cdot \mathrm{L}^{-1}$, respectively. In effluent, the detected concentrations for naproxen, ibuprofen and diclofenac were in the ranges of $0.6-1.1$, 2.1-4.2 and 1.4-2.0 $\mu \mathrm{g} \cdot \mathrm{L}^{-1}$, respectively. Overall, the employed SPE-HPLC method led to rapid pre-concentration of target compounds prior to their trace quantification in wastewater samples.
\end{abstract}

Keywords: solid-phase extraction, wastewater treatment plants, ibuprofen, naproxen, diclofenac

\section{INTRODUCTION}

The presence of pharmaceutical compounds in the environment is a growing concern to analytical chemists and the general public. Pharmaceutical compounds such as naproxen, ibuprofen and diclofenac are useful compounds that belong to the group of non-steroidal anti-inflammatory drugs (NSAIDs). These organic compounds are polar and acidic. Both naproxen and diclofenac have a $\mathrm{pKa}$ value of 4.2 , whereas that of ibuprofen is 4.9 (Table 1) (Dahane et al., 2013). NSAIDs are widely used by humans for the treatment of rheumatoid arthritis (Mahkam and Poorgholy, 2011). Once consumed, they are subjected to human metabolism, followed by excretion in urine and faeces as metabolites and as unaltered parent compounds, which can be subjected to further transformations in wastewater treatment plants (WWTPs) (Parrilla Vazquez et al., 2013).

Overdose or chronic abuse of NSAIDs can lead to toxic side-effects (Lagha et al., 2011). Health effects caused by the consumption of acidic pharmaceuticals by animals at low levels is not understood; however, diclofenac has been reported to be the cause of vulture population declines in Asia (Oaks et al., 2004; Taggart et al., 2007). Diclofenac is also known as a compound that affects organ histology and gene expression in fish at concentrations as low as $1 \mu \mathrm{g} \cdot \mathrm{L}^{-1}$ (Cuklev et al., 2012). Therefore, the development of a very sensitive analytical

To whom all correspondence should be addressed.

e-mail: lawrencem2@dut.ac.za

Received 6 June 2016; accepted in revised form 20 March 2017 methodology for the study of NSAIDs in various sample matrices is required.

In many countries, the removal efficiency for NSAIDs during the wastewater treatment process has been investigated (Lindqvist et al., 2005; Yu et al., 2006; Zorita et al., 2009). For example, in a municipal sewage treatment system located in the south of Sweden, a removal efficiency of $94 \%$ and $99 \%$ for naproxen and ibuprofen, respectively, was reported (Zorita et al., 2009). However, in the same study, diclofenac was not removed during the wastewater treatment process. The removal efficiency for ibuprofen, naproxen and diclofenac in a WWTP located in Germany was $87 \%, 88 \%$ and $18 \%$, respectively (Yu et al., 2006). In WWTPs located in Finland, the removal efficiencies were in the ranges of $78-100 \%, 55-98 \%$ and $9-60 \%$ for ibuprofen, naproxen and diclofenac, respectively (Lindqvist et al., 2005). All these studies show the incomplete removal of such compounds from WWTPs. As a consequence of this, such compounds have also been detected in river water samples from Spain (Carmona et al., 2014). The same authors reported the occurrence of ibuprofen, naproxen, diclofenac and other pharmaceutical compounds at ng. $\mathrm{L}^{-1}$ levels in tap and mineral water samples collected from Spain. The occurrence of pharmaceutical compounds in WWTP effluents, surface water and drinking water samples in Europe demands a detailed screening of such compounds on a worldwide scale.

Although there is enough evidence on the occurrence of NSAIDs in European water bodies (Carmona et al., 2014; Yu et al., 2006; Rodil et al., 2012; Togola and Budzinski, 2007), the presence of such compounds in the South African environment is not fully known. Relatively few published reports on the occurrence of NSAIDs in South African WWTPs have emerged 
(Agunbiade and Moodley, 2014; Agunbiade and Moodley, 2016; Amdany et al., 2014; Amdany et al., 2015; Gumbi et al., 2017; Madikizela and Chimuka, 2016; Matongo et al., 2015a; Matongo et al., 2015b). These published studies report the concentration of selected pharmaceuticals in aqueous samples collected from a few geographical areas of the Gauteng and KwaZulu-Natal provinces of South Africa. For example, most studies reported for KwaZulu-Natal Province focused on environmental pollution in the north of the city of Durban and in locations around Pietermaritzburg (Agunbiade and Moodley, 2014; Agunbiade and Moodley, 2016; Gumbi et al., 2017; Madikizela and Chimuka, 2016; Matongo et al., 2015a; Matongo et al., 2015b). Therefore, more work is required in order to assess the extent of water pollution in several regions of South Africa. This study is based on the determination of selected NSAIDs in Umbilo and Kingsburgh WWTPs. To the best of our knowledge, there are currently no available reports on the NSAID content at these sites. However, the presence of metals at these sites has been reported (Naidoo et al., 2013).

Analytical techniques such as gas and high performance liquid chromatography (HPLC) for trace determination of pharmaceuticals in aqueous samples are well established (Togola and Budzinski, 2007; Rodil et al., 2012). In gas chromatographic analysis, derivatization is employed for the improvement of volatility of target compounds (Gumbi et al., 2017; Rodil et al., 2012; Togola and Budzinski, 2007). Togola and Budzinski (2007) derivatized acidic pharmaceuticals with $N$-Methyl- $N$ (trimethylsilyl)trifluoroacetamide prior to gas chromatographic analysis. In order to avoid a derivatization process which increases the analysis time, a direct injection of samples and compounds into a liquid chromatographic instrument is done (Rodil et al., 2012). A suitable sample preparation technique is employed prior to the chromatographic separation of target compounds. Solid-phase extraction (SPE) technique using Oasis

\begin{tabular}{|l|l|l|l|}
\hline \multicolumn{3}{|c|}{ TABLE 1 } \\
Physicochemical properties for ibuprofen, naproxen and \\
diclofenac (Dahane et al., 2013)
\end{tabular}

HLB sorbent is widely used for pre-concentration of NSAIDs and elimination of interfering species (Agunbiade and Moodley, 2014; Agunbiade and Moodley, 2016; Amdany et al., 2014; Gumbi et al., 2017; Matongo et al., 2015; Matongo et al., 2015). However, such sorbent is most suitable when target compounds exhibit both hydrophilic and lipophilic properties (Madikizela et al., 2014). The current study focused primarily on the occurrence of hydrophilic compounds in wastewater; hence, a solid-phase extraction methodology was developed using Oasis MAX sorbent. Oasis MAX is made of a mixed-mode polymer sorbent with both reversed-phase and anion-exchange functionalities (Lee et al., 2005).

Therefore, the three objectives of this study were to:

- Investigate a suitable sample preparation technique for the extraction and pre-concentration of ibuprofen, naproxen and diclofenac from wastewater

- Study the occurrence of ibuprofen, naproxen and diclofenac in WWTPs located around Durban, KwaZulu-Natal Province, South Africa

- Evaluate the removal efficiency for ibuprofen, naproxen and diclofenac from local WWTPs

\section{MATERIALS AND METHODS}

\section{Materials and reagents}

Naproxen (98\%), ibuprofen ( $\geq 98 \%$ ) and diclofenac sodium salt were purchased from Sigma-Aldrich (Steinheim, Germany). HPLC-grade solvents such as acetonitrile $(\geq 99.9 \%)$ and methanol (99.5\%) were purchased from Merck (Darmstadt, Germany). Formic acid (approx. 98\%) was purchased from Fluka (Steinheim, Germany). Sodium chloride ( $\geq 99.5 \%$ ) and sodium hydroxide pellets were purchased from Associated Chemical Enterprises (Johannesburg, South Africa).

\section{Instrumentation and conditions}

Chromatographic separation was performed on a HPLC system that consisted of an online mobile phase degasser unit (Model: DGU-20A3), $20 \mu \mathrm{L}$ sample loop, pump (Model: LC-20AB), and photo diode array detector (Model: SPD-M20A), all obtained from Shimadzu Corporation (Kyoto, Japan). The mobile phase used consisted of a mixture of acetonitrile and $0.2 \%$ formic acid in water, at a ratio of 60:40 (v:v). A mobile phase flow rate of 0.8 $\mathrm{mL} \cdot \mathrm{min}^{-1}$ was used in this work. Separation was performed on a Lichrospher $\mathrm{C}_{18}$ HPLC column 250 x $4.00 \mathrm{~mm}$ x $5 \mu \mathrm{m}$ obtained from Merck (Darmstadt, Germany). Shimadzu LC solutions software was used for data collection and processing. Photo diode array detection was performed at the wavelengths of 230 $\mathrm{nm}$ for naproxen and $200 \mathrm{~nm}$ for both ibuprofen and diclofenac.

A Bante900P multi-parameter water quality meter was purchased from Bante instruments (Shanghai, China). Prior to its use, the meter was calibrated using the calibration buffers that were provided by the supplier. Thereafter, the meter was used to monitor the physicochemical properties of the wastewater during the sampling.

For SPE, vacuum manifold purchased from Phenomenex (Carlifonia, USA) was connected to a vacuum pump obtained from Pall Corporation (Fribourg, Switzerland). Oasis MAX 6cc $150 \mathrm{mg}$ solid-phase extraction cartridges were obtained from Waters Corporation (Milford, Massachusetts USA). 


\section{Validation of analytical method}

A stock solution (100 mg. $\left.\mathrm{L}^{-1}\right)$ containing all target compounds was prepared in acetonitrile. The solution was then diluted in order to prepare working solutions. Each working solution was analysed using an HPLC system. Limit of detection, limit of quantification and linearity were computed.

Accuracy and precision of the analytical method were determined using deionized water that was spiked with all target compounds at concentration levels of 50 and $5 \mu \mathrm{g} \cdot \mathrm{L}^{-1}$. The spiking concentrations were selected based on the levels of target compounds reported previously in other South African WWTPs (Amdany et al., 2014; Madikizela and Chimuka, 2016; Matongo et al., 2015a). In such studies, the target compounds were detected in wastewater at low $\mu \mathrm{g} \cdot \mathrm{L}^{-1}$ levels. The optimized solid phase extraction method was employed for the extraction and preconcentration of target compounds prior to HPLC quantification.

\section{Sampling}

Wastewater samples were collected weekly from the influent and effluent of Umbilo and Kingsburgh WWTP using precleaned glass bottles in May 2016. Effect of chlorination on target compounds was investigated by collecting samples before and after the disinfection stage. Umbilo and Kingsburgh WWTPs are located in the Province of KwaZulu-Natal in South Africa. The GPS co-ordinates for the location of Umbilo and Kingsburgh WWTPs are S29.84556 ${ }^{\circ} \mathrm{E} 30.89103^{\circ}$ and $\mathrm{S} 30.07445^{\circ} \mathrm{E} 30.85687^{\circ}$, respectively. Physicochemical properties of the samples were measured in situ; thereafter, the samples were transported to the laboratory. Samples were filtered through a $0.22 \mu \mathrm{m}$ nylon syringe filter obtained from Membrane Solutions (Dallas, USA), and stored in the refrigerator $\left(4^{\circ} \mathrm{C}\right)$ until analysis.

\section{Solid-phase extraction}

The solid-phase extraction method was optimized using a standard solution in order to achieve high extraction efficiency for target compounds. Optimized parameters were sample $\mathrm{pH}$, sample volume, elution solvent and the effect of salinity.

Pre-optimized conditions were used to treat the collected wastewater samples. The SPE cartridge was conditioned with $5 \mathrm{~mL}$ of acetonitrile followed by $5 \mathrm{~mL}$ of acidified deionized water ( $\mathrm{pH} 2.5)$ both loaded at a flow rate of $1 \mathrm{~mL} \cdot \mathrm{min}^{-1}$. The acidified ( $\mathrm{pH} 2.5)$ wastewater sample $(100 \mathrm{~mL}$ ) was loaded onto the SPE cartridge at a flow rate of $1 \mathrm{~mL} \cdot \mathrm{min}^{-1}$. The cartridge was washed with $5 \mathrm{~mL}$ of methanol:water (10:90\%, v:v). Thereafter, the retained compounds were eluted sequentially with $2 \mathrm{~mL}$ methanol, $2 \mathrm{~mL}$ mixture of methanol and acetic acid (90:10, $\mathrm{v}: \mathrm{v})$ and $2 \mathrm{~mL}$ of $2 \%(\mathrm{v}: \mathrm{v})$ formic acid diluted using a mixture of methanol and acetic acid (40:60, v:v). The volume of the collected extract was reduced to $0.5 \mathrm{~mL}$ with a gentle stream of nitrogen gas prior to injection into the HPLC system.

\section{Removal efficiency for target compounds from wastewater treatment plants}

The removal efficiency $(R)$ for each compound from both WWTPs was evaluated by employing Eq. 1:

$R=\frac{C_{\text {infl }}-C_{\text {effl }}}{C_{\text {infl }}} \times 100$ where: $C_{i n f l}$ and $C_{e f f l}$ are the concentrations obtained for the raw influent and final effluent, respectively (Sari et al., 2014).

\section{RESULTS AND DISCUSSION}

\section{Optimization of extraction conditions}

\section{Effect of sample pH}

The effect of sample $\mathrm{pH}$ on SPE was investigated by loading 10 $\mathrm{mL}$ of spiked deionized water into the cartridge. The $\mathrm{pH}$ of the spiked deionized water sample was varied in the range of 2.5-11. The results obtained (Fig. 1) indicated that the recoveries for all compounds decreased when the $\mathrm{pH}$ of the water solution was increasing. This was an expected phenomenon for the reversedphase mode. When a water sample is acidified to a $\mathrm{pH}$ that is less than the $\mathrm{pKa}$ value of target compounds given in Table 1, the acids are non-ionized which leads to their adsorption through the reversed-phase interactions (Lee et al., 2005). Therefore, in subsequent experiments, the $\mathrm{pH}$ in all the sample solutions was adjusted to 2.5 .

\section{Effect of elution solvent}

Different solvent conditions were investigated for the elution of the compounds that were adsorbed on the SPE cartridge. The elution solvents investigated were methanol, acetic acid in methanol (10:90, v:v) and 2\% (v:v) formic acid in a mixture of methanol and acetonitrile (40:60, v:v). The results presented in Fig. 2 (a) clearly show that each elution solvent investigated was able to elute a significant amount of a particular compound. The results show that $53 \%$ of naproxen was eluted with methanol. The best elution solvent conditions for diclofenac, which yielded $53 \%$ and $54 \%$, were acetic acid in methanol (10:90, v:v) and $2 \%$ (v:v) formic acid in a mixture of methanol and acetonitrile $(40: 60, v: v)$. Furthermore, $60 \%$ of ibuprofen was eluted with $2 \%(\mathrm{v}: \mathrm{v})$ formic acid in a mixture of methanol and acetonitrile (40:60, v:v). Therefore, a sequential elution was employed, in which all the investigated solvents were used in series. It has been documented (Lee et al., 2005) that the elution of compounds retained in Oasis MAX cartridge with methanol only removes the less acidic compounds, alongside other neutral co-extracts which were mainly adsorbed by reversed-phase mechanism. More acidic compounds are usually eluted with $2 \%$ formic acid mixture in the non-ionized form (Lee et al., 2005).

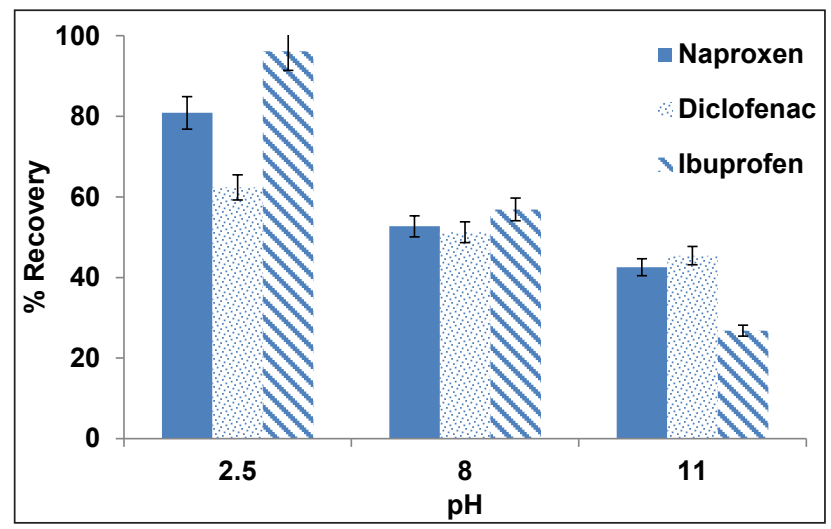

Figure 1

Effect of sample $\mathrm{pH}$ on solid phase extraction 
In this study the eluted fractions were further combined and evaporated with a gentle steam of nitrogen to $0.5 \mathrm{~mL}$. Results in Fig. 2 (b) show that the recoveries of $74 \%, 85 \%$ and $97 \%$ were achieved for naproxen, diclofenac and ibuprofen, respectively.

\section{Effect of salt concentration}

The effect of salt content was investigated by spiking a mixture of target compounds in deionized water with different amounts of sodium chloride. The $\mathrm{pH}$ of each spiked solution was adjusted to 2.5. Thereafter, $100 \mathrm{~mL}$ of each solution was loaded into a preconditioned SPE cartridge. Therefore, percentage recovery was determined for each target compound. Addition of salt to the aqueous samples is usually carried out to improve the extraction of several compounds (Sarafraz-Yazdi et al., 2012). However, in this study, salt was added in pure compounds to imitate the wastewater contents. Results presented in Fig. 3 show a decrease in per cent recovery as the concentration of sodium chloride increases from 0.1 to $0.4 \%(\mathrm{~m}: \mathrm{v})$. This trend has also been observed elsewhere ( $\mathrm{Li}$ et al., 2008), where the adsorption amounts of three phthalic acid esters decreased with increasing ionic strength. Sodium chloride dissociates in water, after which competition of sodium ions with target compounds for adsorption sites is expected to occur. This could lead to occupation of the adsorbent surface by sodium ions which could lead to the unavailability of sites for the adsorption of target compounds.

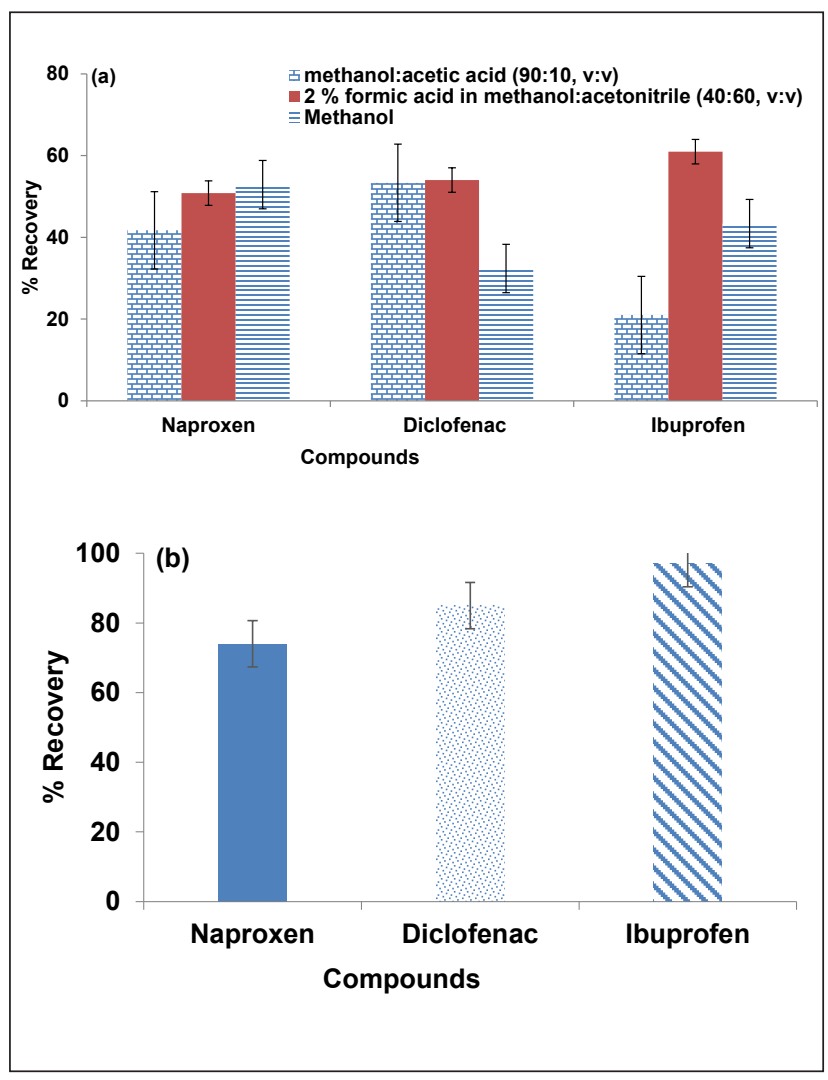

Figure 2

Effect of elution solvent on solid phase extraction, where (a) shows the $\%$ recovery after elution with individual solvents and (b) shows the percentage recoveries obtained after sequential elution of target compounds from SPE cartridge using $2 \mathrm{~mL}$ each of methanol, acetic acid in methanol (10:90, v:v) and 2\% (v:v) formic acid in a mixture of methanol and acetonitrile (40:60, v:v); followed by evaporation of $6 \mathrm{~mL}$ of eluate to $0.5 \mathrm{~mL}$.

\section{Effect of sample volume}

Different volumes of deionized water that was spiked with naproxen, ibuprofen and diclofenac at $\mathrm{pH} 2.5$ were loaded into a pre-conditioned SPE cartridge. Per cent recovery was determined in each case. Results in Fig. 4 indicate that the per cent recoveries increased when the sample volume was increased. For instance, the recovery for $10 \mathrm{~mL}$ was low, perhaps due to limited sample interaction with sorbent as not enough volume was available to push the whole sample through the cartridge volume. Sample volume was not increased beyond 100 $\mathrm{mL}$ as this would have resulted in a long analysis time. However, in our previous work it was shown that per cent recoveries tend to decrease when loading large sample volumes into the SPE cartridge (Madikizela et al., 2014). This happens when the capacity of the SPE sorbent has been exceeded and therefore the cartridge becomes overloaded.

\section{Method validation}

The separation of target compounds was achieved on the reverse phase separation column (Fig. 5). The performance of the high performance liquid chromatographic method was validated by determining the limit of detection (LOD), limit of quantification (LOQ) and linearity. LOD and LOQ were determined experimentally for each compound where the detectable amounts showed a signal-to-noise ratio of 3 and 10 , respectively. The HPLC instrument employed in this study was able to detect up to $0.1 \mu \mathrm{g} \cdot \mathrm{L}^{-1}$ for ibuprofen and diclofenac, whereas $0.04 \mu \mathrm{g} \cdot \mathrm{L}^{-1}$ was observed for naproxen. LOQ values of

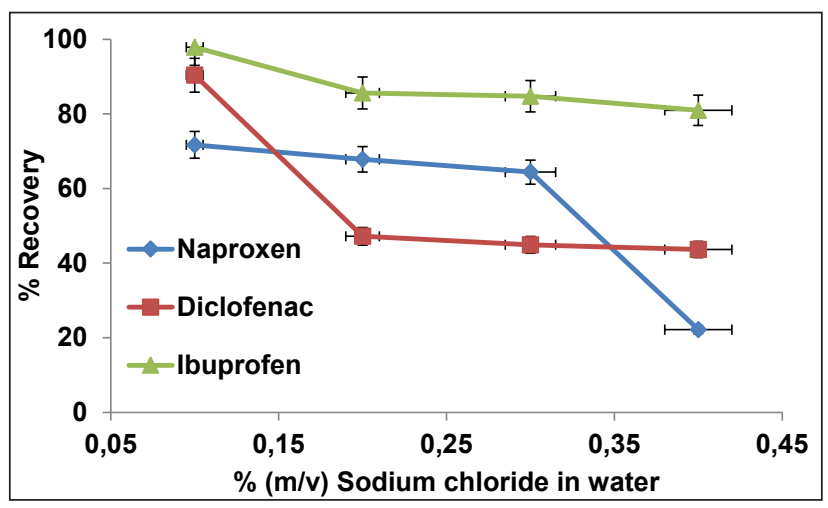

Figure 3

Effect of salt content on solid phase extraction

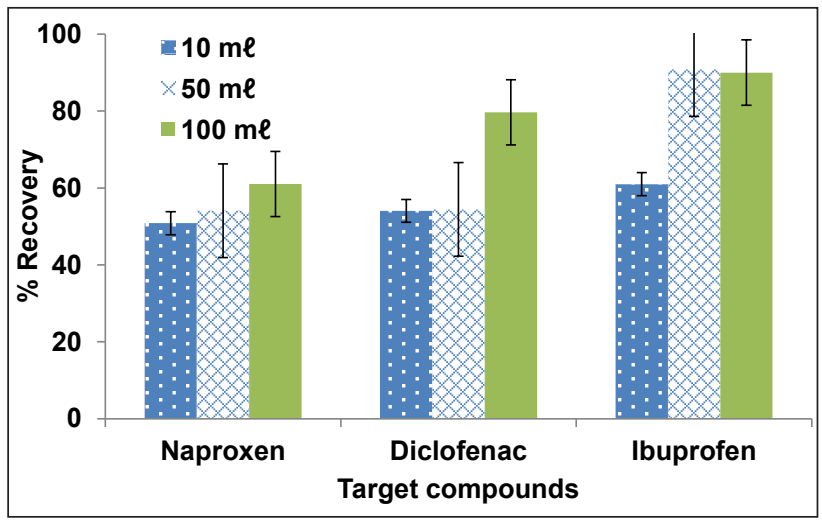

Figure 4

Effect of sample volume on solid phase extraction 
0.4, 0.1 and $0.4 \mu \mathrm{g} \cdot \mathrm{L}^{-1}$ were obtained for ibuprofen, naproxen and diclofenac, respectively. The LODs obtained (Table 2) were lower than those reported in literature for the analysis of the same compounds using photo diode array detection, which in turn means that the proposed method is more sensitive (Payan et al., 2011). Calibration curves were linear for all of the compounds with correlation coefficients over 0.99 .

Furthermore, the sample preparation method was validated by determining the per cent recoveries when the optimized SPE conditions were employed for the deionized water that was spiked with all target compounds at 50 and $5 \mu \mathrm{g} \cdot \mathrm{L}^{-1}$. Acceptable per cent recoveries as indicated in Table 2 were obtained with good precision for all compounds. These results indicate that the sample preparation method proposed in this study was suitable for the extraction and pre-concentration of ibuprofen, naproxen and diclofenac. After successful validation of the SPE-HPLC method in deionized water, the same protocol was applied to the analysis of wastewater samples.

\section{Environmental analysis}

The optimized SPE conditions were applied for the quantitative analysis of ibuprofen, naproxen and diclofenac in wastewater collected from Kingsburgh and Umbilo WWTPs. The treatment process in both plants involves screening for grit removal, settling tanks for sludge production and removal, aeration tanks and chlorination for disinfection. Compounds of interest present in wastewater were identified by using retention time comparison in conjunction with photo diode array detection

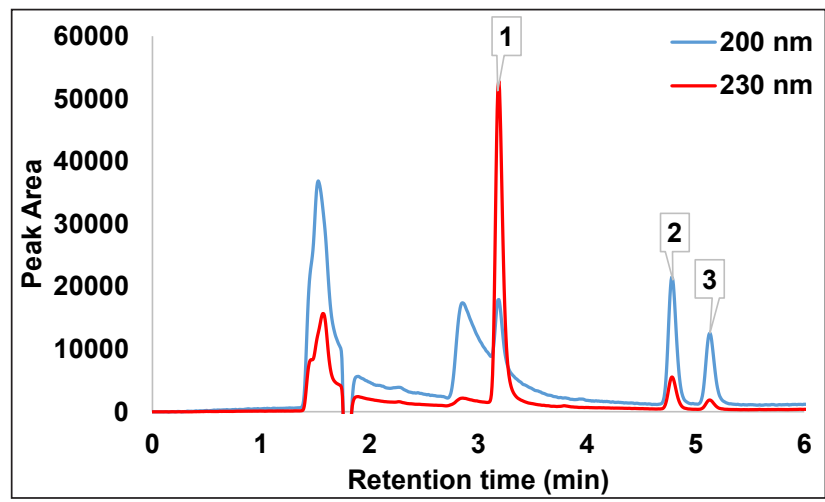

Figure 5

Chromatograms obtained for the separation of $1000 \mu \mathrm{g} \cdot \mathrm{L}^{-1}$ of ibuprofen, naproxen and diclofenac. Peaks 1, 2 and 3 represent naproxen, diclofenac and ibuprofen.

TABLE 2

Limit of detection, limit of quantification, correlation coefficient and per cent recovery for ibuprofen, naproxen and diclofenac

\begin{tabular}{|c|c|c|c|c|c|}
\hline \multirow{2}{*}{ Compound } & \multirow{2}{*}{$\begin{array}{c}\text { LOD } \\
\left(\mu \mathrm{g} \cdot \mathrm{L}^{-1}\right)\end{array}$} & \multirow{2}{*}{$\begin{array}{c}\text { LOQ } \\
\left(\mu g \cdot L^{-1}\right)\end{array}$} & \multirow{2}{*}{$\mathbf{R}^{2}$} & \multicolumn{2}{|c|}{$\begin{array}{c}\text { \% Recovery } \pm \\
\% R S D\end{array}$} \\
\hline & & & & $\begin{array}{c}50 \\
\mu \mathrm{g} \cdot \mathrm{L}^{-1}\end{array}$ & $5 \mu \mathrm{g} \cdot \mathrm{L}^{-1}$ \\
\hline Ibuprofen & 0.13 & 0.42 & 0.995 & $94 \pm 1.4$ & $95 \pm 0.9$ \\
\hline Naproxen & 0.04 & 0.12 & 0.995 & $76 \pm 2.7$ & $83 \pm 11$ \\
\hline Diclofenac & 0.12 & 0.39 & 0.994 & $81 \pm 4.3$ & $98 \pm 0.3$ \\
\hline
\end{tabular}

spectra. The collected data are summarized in Figs. 5-8, and Figs. A1-A3 in Appendix 1. The presence of target compounds was observed at each stage of the wastewater treatment process (Figs. 6-8). The chromatograms in Fig. 8 clearly show the incomplete removal of target compounds during the wastewater treatment process. The results (Table 3 ) further showed that disinfection with chlorine does not really reduce the amounts of target compounds in wastewater, except in the case of diclofenac in Umbilo WWTP, which is in agreement with the results reported elsewhere (Behera et al., 2011). This could be due to the fact that the purpose of disinfection is to reduce pathogenic microorganisms which do not have an effect on the quantity of the target compounds present in wastewater (Behera et al., 2011).

Concentration levels obtained for target compounds in wastewater (Table 3) were comparable to those reported in other studies. For example, mean concentrations for ibuprofen in the influent of Goudkoppies and Northern WWTPs located in Johannesburg, South Africa, were 39.8 and $111.9 \mu \mathrm{g} \cdot \mathrm{L}^{-1}$, respectively (Amdany et al., 2014). In another study conducted in the north of Spain, ibuprofen and naproxen concentration in the influent ranged from $2.3-42 \mu \mathrm{g} \cdot \mathrm{L}^{-1}$, whereas a maximum concentration of $5.7 \mu \mathrm{g} \cdot \mathrm{L}^{-1}$ was reported for the effluent (Fernandez et al., 2014). Few studies that have been conducted on wastewater have shown the presence of pharmaceuticals in WWTPs located around the city of Durban, South Africa (Agunbiade and Moodley, 2014; Madikizela et al., 2014; Madikizela and Chimuka, 2016; Matongo et al., 2014). The extent of water pollution is not fully understood in South Africa; hence, future studies should focus on the monitoring of naproxen, ibuprofen and diclofenac in surface water, aquatic plants and sediments.

The removal efficiency for each compound in WWTPs was determined based on the raw influent and final effluent concentrations. As shown in Table 3, the percentage removal for target compounds in both WWTPs was in the range of 69-97\%. Diclofenac had the lowest percentage removal from Umbilo WWTP, with 69\% removal efficiency. In the same WWTP, the removal efficiency observed for naproxen and ibuprofen exceeded 90\%. The highest removal efficiency (97\%) for both naproxen and ibuprofen was observed in Kingsburgh WWTP. In Kingsburgh WWTP, the removal efficiency for diclofenac was $92 \%$. These results corresponded well with figures reported for another Durban WWTP (Agunbiade and Moodley, 2014). In general, Umbilo and Kingsburgh WWTPs showed a better removal efficiency when compared to some other wastewater treatment works worldwide. For instance, the removal efficiencies reported for ibuprofen, naproxen and diclofenac in a WWTP located in Baltimore, USA, were $87 \%, 88 \%$ and $18 \%$, respectively (Yu et al., 2006). Removal efficiencies greater than $90 \%$ for naproxen and ibuprofen have been reported for a WWTP that uses UV for disinfection; however, the same plant did not show any removal for diclofenac (Carmona et al., 2014). Poor removal of diclofenac has been reported for a number of WWTPs (Lindqvist et al., 2005; Rosal et al., 2010; Zorita et al., 2009; Samaras et al., 2013). Further work is required in order to investigate the effect of poor removal and to rectify the problem for diclofenac. Such work could involve the investigation of the ability of diclofenac to partition between wastewater and sludge. Although the studied WWTPs were not specifically designed to remove pharmaceutical compounds, they showed a great potential in this regard.

In general, the performance of the studied WWTPs was evaluated by monitoring $\mathrm{pH}$, conductivity, total dissolved solids, dissolved oxygen, salinity and oxidation reduction potential. The results (Table S1) indicated normal functioning of the WWTPs. This observation was based on the reduction of conductivity, 


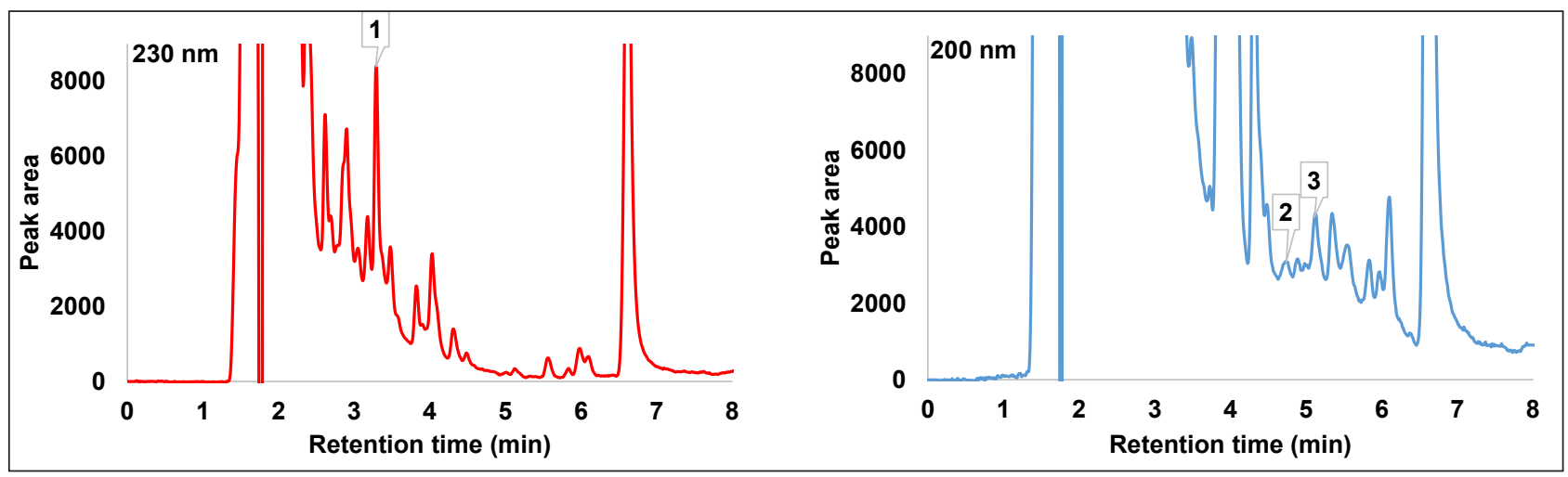

Figure 6

Chromatograms for raw wastewater influent recorded at 200 and $230 \mathrm{~nm}$; Peaks 1, 2 and 3 represent naproxen, diclofenac and ibuprofen

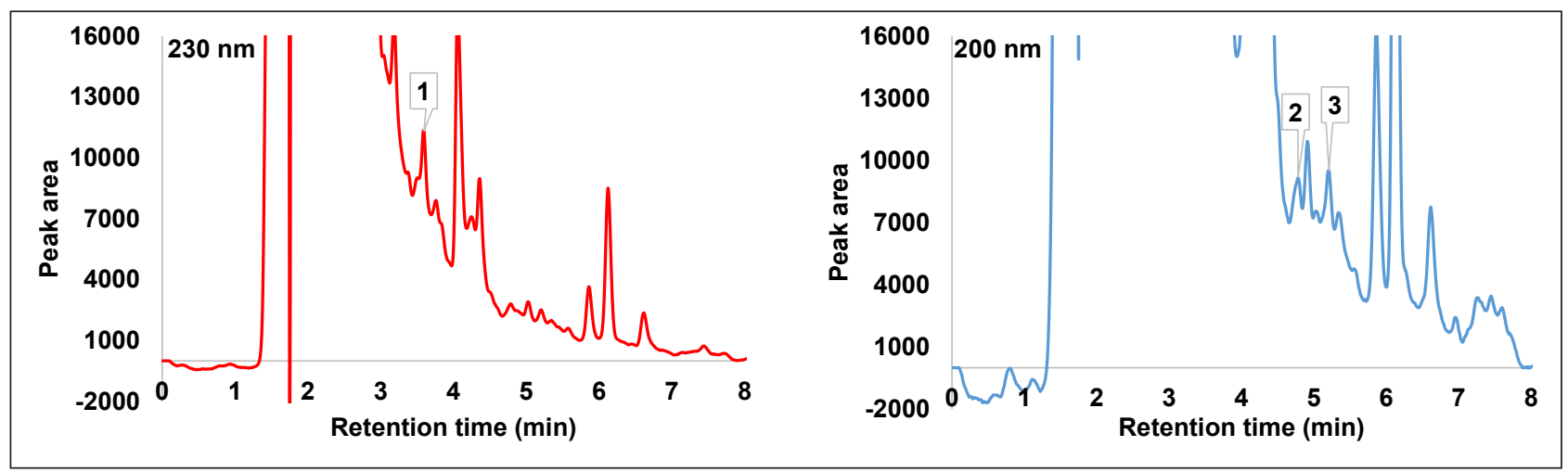

Figure 7

Chromatograms for wastewater samples collected prior to chlorination; Peaks 1, 2 and 3 represent naproxen, diclofenac and ibuprofen

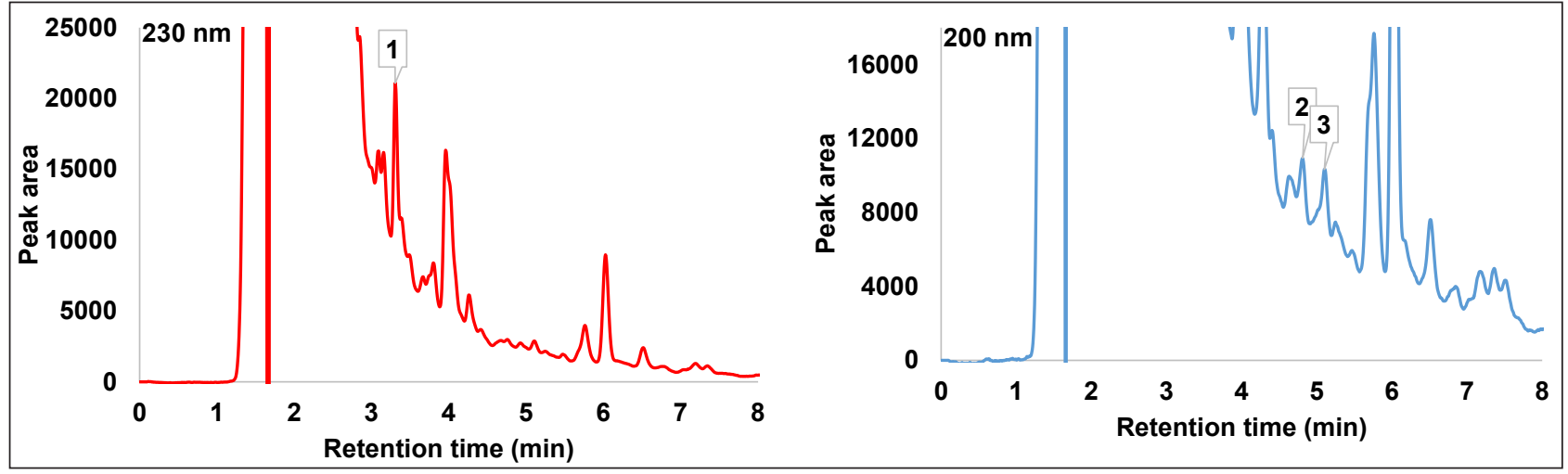

Figure 8

Chromatograms for wastewater effluent recorded at 200 and $230 \mathrm{~nm}$; Peaks 1, 2 and 3 represent naproxen, diclofenac and ibuprofen

TABLE 3

Quantities $\left(\mu \mathrm{g} \cdot \mathrm{L}^{-1}\right)$ of target compounds \pm standard deviation $(n=3)$ detected in WWTPs and removal efficiency $(\%)$

\begin{tabular}{|l|c|c|c|c|c|c|c|c|}
\hline & \multicolumn{3}{|c|}{ Kingsburgh WWTP } & \multicolumn{5}{c|}{ Umbilo WWTP } \\
\hline Compound & Influent & Mid & Effluent & $\% R$ & Influent & Mid & Effluent & $\%$ R \\
\hline Ibuprofen & $69 \pm 6.5$ & $1.9 \pm 1.6$ & $2.1 \pm 2.9$ & 97 & $55 \pm 6.6$ & $5.0 \pm 1.3$ & $4.2 \pm 1.0$ & 92 \\
\hline Naproxen & $20 \pm 13$ & $0.9 \pm 0.6$ & $0.6 \pm 0.7$ & 97 & $15 \pm 11$ & $1.3 \pm 0.8$ & $1.1 \pm 0.7$ & 93 \\
\hline Diclofenac & $16 \pm 6.5$ & $1.7 \pm 1.2$ & $1.4 \pm 1.1$ & 92 & $6.4 \pm 2.7$ & $5.5 \pm 4.9$ & $2.0 \pm 0.6$ & 69 \\
\hline
\end{tabular}

Mid: sample collected prior to disinfection, \%R: Removal efficiency 
salinity and total dissolved solids from the raw influent to the final effluent. In addition, the increase in dissolved oxygen from the influent to the effluent was an indication of a functional WWTP.

\section{CONCLUSIONS}

The application of advanced HPLC equipped with photo diode array detector for environmental analyses has allowed for the separation and quantification of ibuprofen, naproxen and diclofenac in wastewater. In this study, the occurrence of naproxen, ibuprofen and diclofenac in Umbilo and Kingsburgh WWTPs located in Durban has been reported. Solid-phase extraction method with Oasis MAX offered improved results since the target compounds were extracted and preconcentrated. Acceptable recoveries that ranged from $76 \%$ to $98 \%$ were obtained for the target compounds. All compounds were detected in the raw influent and effluent.

Neither WWTP was specifically designed for removal of pharmaceutical compounds: however, the per cent removal shows that it is possible to remove the drugs to some extent during the treatment process. Therefore, it is recommended to optimize the design of the WWTPs for effective removal of such drugs during the wastewater treatment process. In order to achieve this, the degradation patterns of such acidic pharmaceuticals in wastewater needs to be monitored. Also, the factors that could influence the partitioning of naproxen, ibuprofen and diclofenac between the wastewater and sludge need to be investigated.

\section{ACKNOWLEDGEMENTS}

This work is based on the research supported in part by the National Research Foundation (NRF) of South Africa, Unique Grant Numbers 93986 and 98279. Lulekiwe Mbuyisa’s involvement in SPE optimization is greatly appreciated.

\section{REFERENCES}

AGUNBIADE FO and MOODLEY B (2014) Pharmaceuticals as emerging organic contaminants in Umgeni River water system, KwaZulu-Natal, South Africa. Environ. Monit. Assess. 186 7273-7291. https://doi.org/10.1007/s10661-014-3926-z

AGUNBIADE FO and MOODLEY B (2016) Occurrence and distribution pattern of acidic pharmaceuticals in surface water, wastewater, and sediment of the Msunduzi River, KwaZuluNatal, South Africa. Environ. Toxicol. Chem 35 36-46. https://doi. org/10.1002/etc.3144

AMDANY R, CHIMUKA L and CUKROWSKA E (2014) Determination of naproxen, ibuprofen, and triclosan in wastewater using the polar organic chemical integrative sampler (POCIS): A laboratory calibration and field application. Water SA 40 407-414. https://doi.org/10.4314/wsa.v40i3.3

AMDANY R, MOYA A, CUKROWSKA E and CHIMUKA L (2014) Optimization of the temperature for the extraction of pharmaceuticals from wastewater by a hollow fiber silicone membrane. Anal. Lett. 48 2343-2356. https://doi.org/10.1080/000327 19.2015.1033722

BEHERA SK, KIM HW, OH J and PARK H (2011) Occurrence and removal of antibiotics, hormones and several other pharmaceuticals in wastewater treatment plants of the largest industrial city of Korea. Sci. Total Environ. 409 4351-4360. https://doi.org/10.1016/j. scitotenv.2011.07.015

CARMONA E, ANDREU V and PICO Y (2014) Occurrence of acid pharmaceuticals and personal care products in Turia River Basis: From waste to drinking water. Sci. Total Environ. 484 53-63. https:// doi.org/10.1016/j.scitotenv.2014.02.085
CUKLEV F, FICK J, CVIJOVIC M, KRISTIANSSON E, FORLIN L and JOAKIM LARSSON DG (2012) Does ketoprofen or diclofenac pose the lowest risk to fish? J. Hazardous Mater. 229-230 100-106. https:// doi.org/10.1016/j.jhazmat.2012.05.077

DAHANE S, GIL GARCIA MD, MARTINEZ BUENO MJ, UCLES MORENO A, MARTINEZ GALERA $M$ and DERDOUR A (2013) Determination of drugs in river and wastewaters using solid-phase extraction by packed multi-walled carbon nanotubes and liquid chromatography-quadrupole-linear ion trap-mass spectrometry. J. Chromatogr. A 1297 17-28. https://doi.org/10.1016/j. chroma.2013.05.002

FERNANDEZ M, FERNANDEZ M, LACA A, LACA A and DIAZ M (2014) Seasonal occurrence and removal of pharmaceutical products in municipal wastewaters. J Environ. Chem. Eng. 2 495-502. https:// doi.org/10.1016/j.jece.2014.01.023

GUMBI BP, MOODLEY B, BIRUNGI G and NDUNGU PG (2017) Detection and quantification of acidic drug residues in South African surface water using gas chromatography-mass spectrometry. Chemosphere 168 1042-1050. https://doi.org/10.1016/j. chemosphere.2016.10.105

LAGHA A, ADHOUM N and MONSER L (2011) A molecularly imprinted polymer for the selective solid-phase extraction of ibuprofen from urine samples. OCBMJ 4 7-13. https://doi. org/10.2174/1875038901004010007

LEE H, PEART TE and SVOBODA ML (2005) Determination of endocrine-disrupting phenols, acidic pharmaceuticals, and personal-care products in sewage by solid-phase extraction and gas chromatography-mass spectrometry. J. Chromatogr. A 1094 122-129. https://doi.org/10.1016/j.chroma.2005.07.070

Li J, CAI Y, SHI Y, MOU S and JIANG G (2008) Analysis of phthalates via HPLC-UV in environmental water samples after concentration by solid-phase extraction using ionic liquid mixed hemimicelles. Talanta 74 498-504. https://doi.org/10.1016/j.talanta.2007.06.008

LINDQVIST N, TUHKANEN T and KRONBERG L (2005) Occurrence of acidic pharmaceuticals in raw and treated sewages and in receiving waters. Water Res. 39 2219-2228. https://doi.org/10.1016/j. watres.2005.04.003

MADIKIZELA LM and CHIMUKA L (2016) Determination of ibuprofen, naproxen and diclofenac in aqueous samples using a multi-template molecularly imprinted polymer as selective adsorbent for solid-phase extraction. J. Pharm. Biomed. Anal. 128 210-215.

MADIKIZELA LM, MUTHWA SF and CHIMUKA L (2014) Determination of triclosan and ketoprofen in river water and wastewater by solid-phase extraction and high performance liquid chromatography. S. Afr. J. Chem. 67 143-150. https://doi. org/10.1016/j.jpba.2016.05.037

MAHKAM M and POORGHOLY N (2011) Imprinted polymers as drug delivery vehicles for anti-inflammatory drugs. Nat. Sci. 9 163-168.

MATONGO S, BIRUNGI G, MOODLEY B and NDUNGU P (2015a) Occurrence of selected pharmaceuticals in water and sediment of Umgeni River, KwaZulu-Natal, South Africa. Environ. Sci. Pollut. Res. 22 10298-10308. https://doi.org/10.1007/s11356-015-4217-0

MATONGO S, BIRUNGI G, MOODLEY B and NDUNGU P (2015b) Pharmaceutical residues in water and sediment of Msunduzi River, KwaZulu-Natal, South Africa. Chemosphere 134 133-140. https://doi. org/10.1016/j.chemosphere.2015.03.093

NAIDOO S, VOSLOO D and SCHOEMAN MC (2013) Foraging at wastewater treatment works increases the potential for metal accumulation in an urban adapter, the banana bat (Neoromicia nana). Afr. Zool. 48 39-55. https://doi.org/10.1080/15627020.2013.11407567

OAKS JL, GILBERT M, VIRANI MZ, WATSON RT, METEYER CU, RIDEOUT BA, SHIVAPRASAD HL, AHMED S, CHAUDHRY MJI, ARSHAD M, MAHMOOD S, ALI A and KHAN AA (2004) Diclofenac residues as the cause of vulture population decline in Pakistan. Nature 427 630-633. https://doi.org/10.1038/nature02317 PARRILLA VAZQUEZ MM, PARRILLA VAZQUEZ P, MARTINEZ GALERA M, GIL GARCIA MD and UCLES A (2013) Ultrasoundassisted ionic liquid dispersive liquid-liquid microextraction coupled with liquid chromatography-quadrupole-linear ion trapmass spectrometry for simultaneous analysis of pharmaceuticals in wastewaters. J. Chromatogr. A 1291 19-26. https://doi.org/10.1016/j. chroma.2013.03.066 
PAYAN MR, LOPEZ MAB, TORRES RF, NAVARRO MV and MOCHON MC (2011) Electromembrane extraction (EME) and HPLC determination of non-steroidal anti-inflammatory drugs (NSAIDs) in wastewater samples. Talanta 85 394-399. https://doi. org/10.1016/j.talanta.2011.03.076

RODIL R, QUINTANA JB, CONCHA-GRANA E, LOPEZ-MAHIA P, MUNIATEGUI-LORENZO S and PRADA-RODRIGUEZ D (2012) Emerging pollutants in sewage, surface and drinking water in Galicia (NW Spain). Chemosphere 86 1040-1049. https://doi.org/10.1016/j. chemosphere.2011.11.053

ROSAL R, RODRIGUEZ A, PERDIGON-MELON JA, PETRE A, GARCIA-CALVO E, GOMEZ MJ, AGUERA A and FERNANDEZALBA AR (2010) Occurrence of emerging pollutants in urban wastewater and their removal through biological treatment followed by ozonation. Water Res. 44 578-588. https://doi.org/10.1016/j. watres.2009.07.004

SAMARAS, VG, STASINAKIS AS, MAMAIS D, THOMAIDIS NS and LEKKAS TD (2013) Fate of selected pharmaceuticals and synthetic endocrine disrupting compounds during wastewater treatment and sludge anaerobic digestion. J. Hazardous Mater. 244-245 259-267. https://doi.org/10.1016/j.jhazmat.2012.11.039

SARAFRAZ-YAZDI A, AMIRI A, ROUNAGHI G and ESHTIAGHHOSSEINI H (2012) Determination of non-steroidal antiinflammatory drugs in water samples by solid-phase microextraction based sol-gel technique using poly(ethylene glycol) grafted multi-walled carbon nanotubes coated fiber. Anal. Chim. Acta 720 134-141. https://doi.org/10.1016/j.aca.2012.01.021

SARI S, OZDEMIR G, YANGIN-GOMEC C, ZENGIN GE, TOPUZ E, AYDIN E, PEHLIVANOGLU-MANTAS E and TAS DO (2014) Seasonal variation of diclofenac concentration and its relation with wastewater characteristics at two municipal wastewater treatment plants in Turkey. J. Hazardous Mater. 272 155-164. https://doi. org/10.1016/j.jhazmat.2014.03.015

TAGGART MA, CUTHBERT R, DAS D, SASHIKUMAR C, PAIN DJ, GREEN RE, FELTRER Y, SHULTZ S, CUNNINGHAM AA and MEHARG AA (2007) Diclofenac disposition in Indian cow and goat with reference to Gyps vulture population declines. Environ. Pollut. 147 60-65. https://doi.org/10.1016/j.envpol.2006.08.017

TOGOLA A and BUDZINSKI H (2007) Analytical development for analysis of pharmaceuticals in water samples by SPE and GC-MS. Anal. Bioanal. Chem. 388 627-635. https://doi.org/10.1007/ s00216-007-1251-x

YU JT, BOUWER EJ and COELHAN M (2006) Occurrence and biodegradability studies of selected pharmaceuticals and personal care products in sewage effluent. Agric. Water Manage. 86 72-80. https://doi.org/10.1016/j.agwat.2006.06.015

ZORITA S, MARTENSSON L and MATHIASSON L (2009) Occurrence and removal of pharmaceuticals in a municipal sewage treatment system in the south of Sweden. Sci. Total Environ. 407 2760-2770.

\section{APPENDIX 1}

TABLE A1

Physicochemical properties measured in wastewater treatment plants. Standard deviations $(n=3)$ are given in \pm values

\begin{tabular}{|c|c|c|c|c|c|c|}
\hline Sample & pH & \begin{tabular}{|c|}
$\begin{array}{c}\text { Conductivity } \\
(\mu \mathrm{s} \cdot \mathrm{cm}-1)\end{array}$ \\
\end{tabular} & $\begin{array}{c}\text { DO } \\
(\mathrm{mg} \cdot \mathrm{L}-1)\end{array}$ & $\begin{array}{c}\text { Salinity } \\
\text { (psu) }\end{array}$ & $\begin{array}{c}\text { TDS } \\
(\mathrm{mg} \cdot \mathrm{L}-1)\end{array}$ & $\begin{array}{c}\text { ORP } \\
\text { (RmV) }\end{array}$ \\
\hline Kingsburgh WWTP influent & $7.10 \pm 0.45$ & $965 \pm 176$ & $0.44 \pm 0.35$ & $0.47 \pm 0.08$ & $477 \pm 82$ & $-29.7 \pm 8.8$ \\
\hline Kingsburgh WWTP prior to disinfection & $7.28 \pm 0.30$ & $599 \pm 27$ & $3.40 \pm 0.42$ & $0.29 \pm 0.01$ & $300 \pm 14$ & $-28.7 \pm 3.3$ \\
\hline Kingsburgh WWTP effluent & $7.39 \pm 0.25$ & $589 \pm 18$ & $5.12 \pm 0.69$ & $0.29 \pm 0.02$ & $299 \pm 10$ & $-29.9 \pm 2.1$ \\
\hline Umbilo WWTP influent & $7.42 \pm 0.26$ & $862 \pm 50$ & $0.45 \pm 0.22$ & $0.42 \pm 0.03$ & $421 \pm 34$ & $-30.2 \pm 6.4$ \\
\hline Umbilo WWTP prior to disinfection & $7.22 \pm 0.43$ & $780 \pm 78$ & $3.34 \pm 0.57$ & $0.39 \pm 0.05$ & $400 \pm 47$ & $-24.9 \pm 4.7$ \\
\hline Umbilo WWTP effluent & $7.13 \pm 0.41$ & $785 \pm 75$ & $5.42 \pm 0.85$ & $0.38 \pm 0.03$ & $395 \pm 37$ & $-25.1 \pm 7.3$ \\
\hline
\end{tabular}

DO - dissolved oxygen; TDS - total dissolved solids; ORP - oxidation reduction potential. 


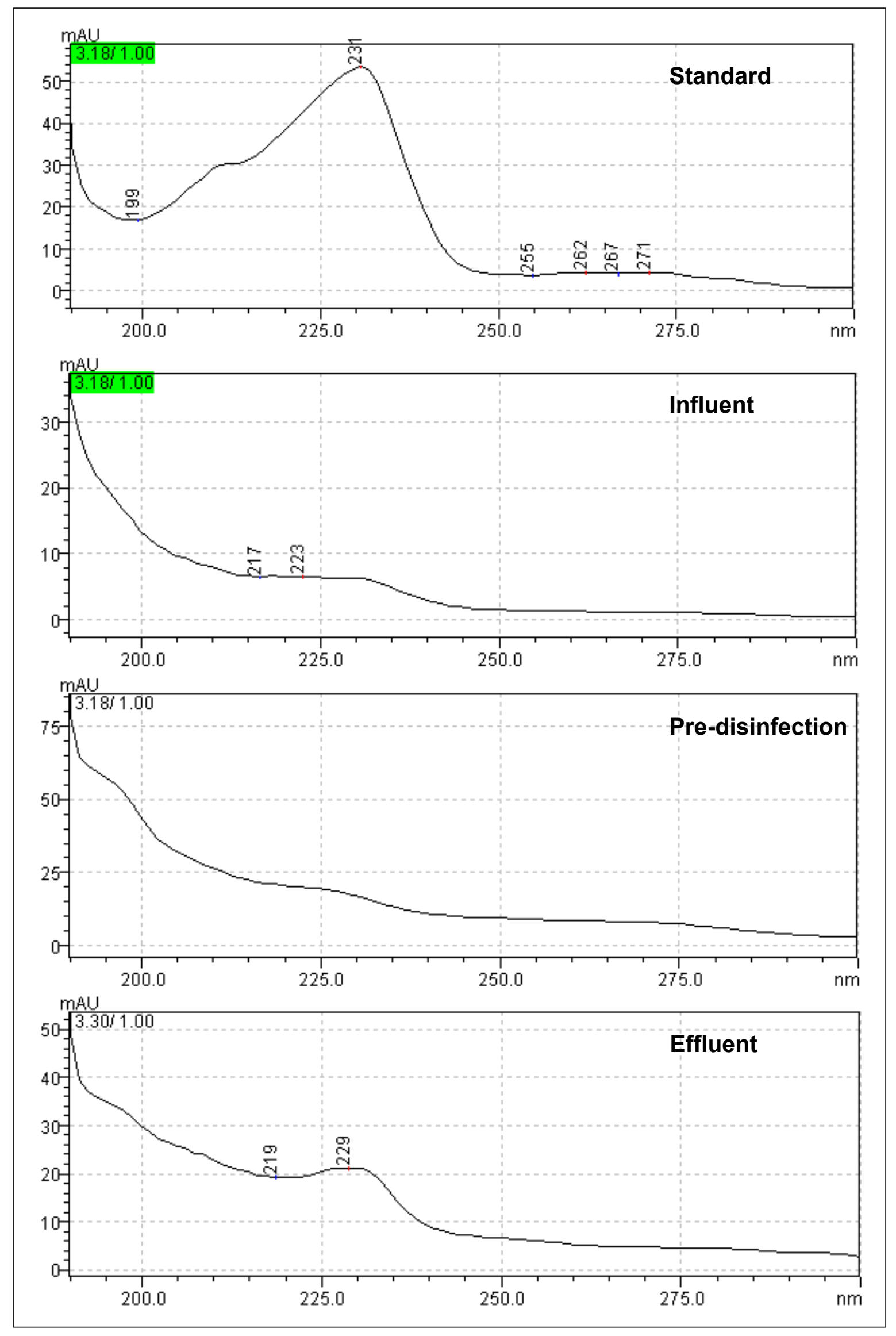

Figure A1

PDA spectra obtained for naproxen in standard solution and in extracted wastewater samples 


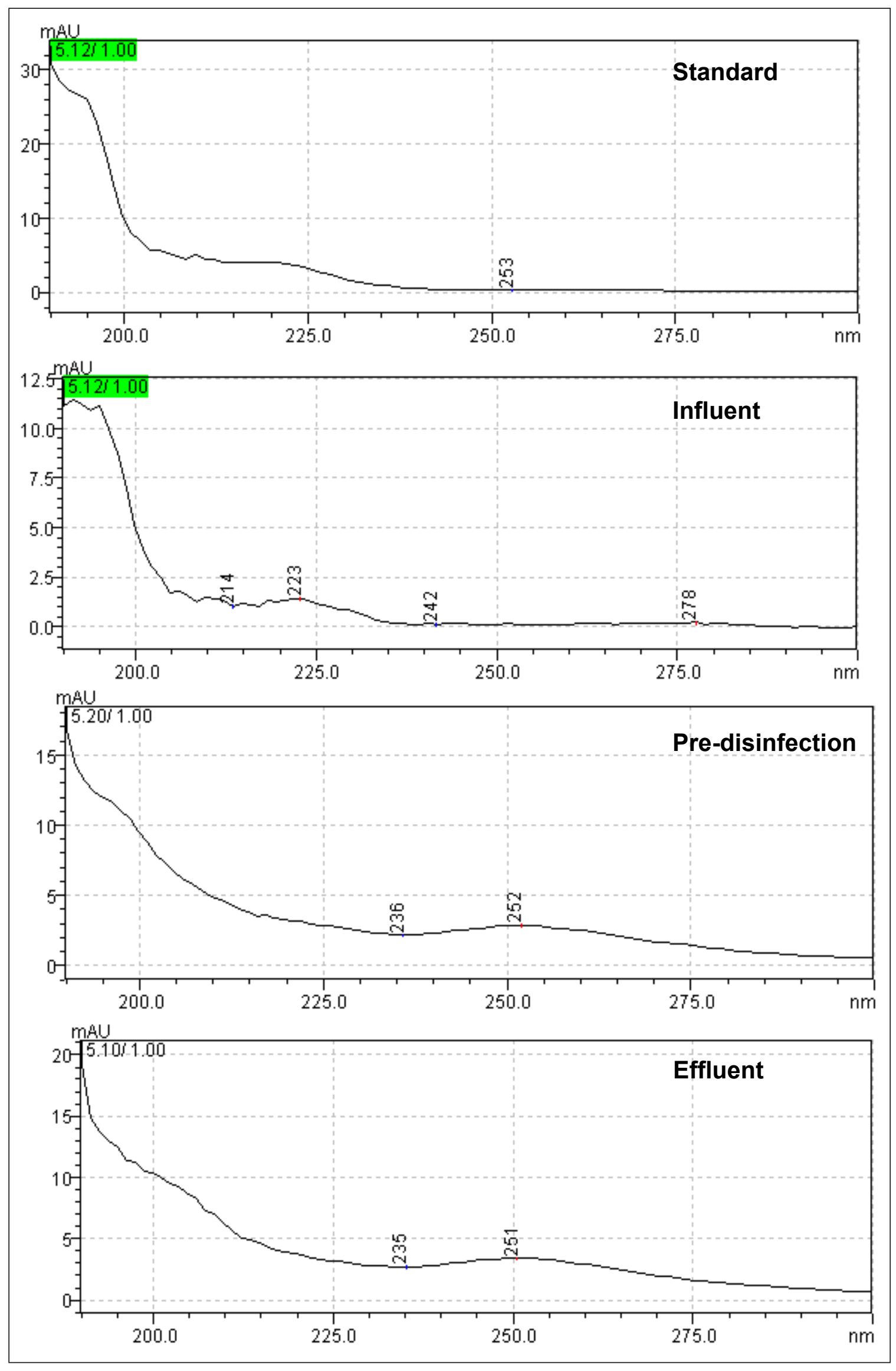

Figure A2

PDA spectra obtained for ibuprofen in standard solution and in extracted wastewater samples 


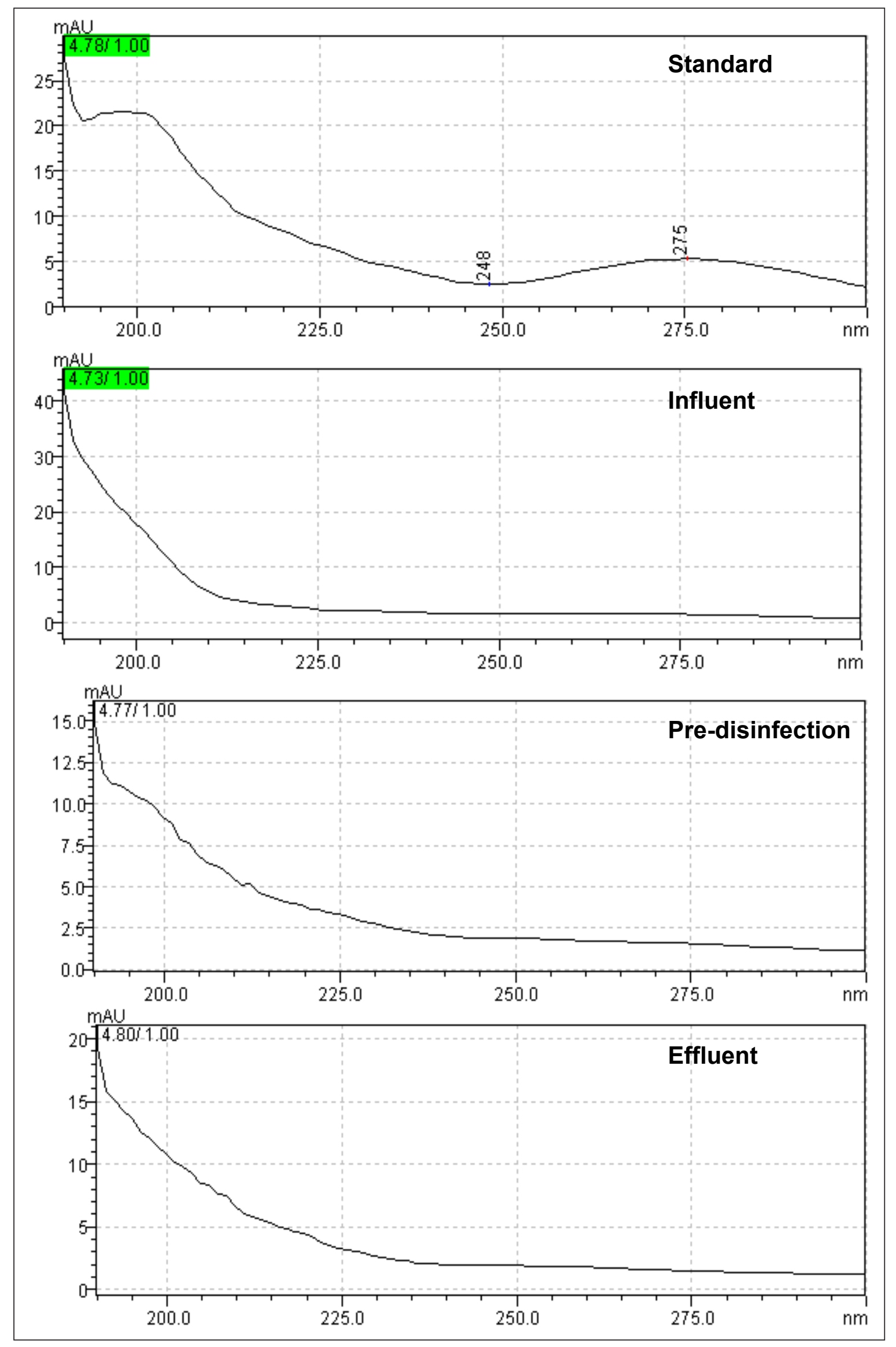

Figure A3

PDA spectra obtained for diclofenac in standard solution and in extracted wastewater samples 